\title{
ANÁLISE DA RESISTÊNCIA E ELASTICIDADE EM FLEXÃO ESTÁTICA EM PAINÉIS LVL DE Eucalyptus grandis PRODUZIDOS COM LÂMINAS PRÉ-CLASSIFICADAS
}

\author{
Nabor da Silveira Pio ${ }^{1}$, Sidon Keinert Júnior ${ }^{2}$, Setsuo Iwakiri ${ }^{2}$, Ulisses Silva da Cunha ${ }^{3}$, \\ Márcio Pereira da Rocha ${ }^{2}$, Fernando Cardoso Lucas Filho ${ }^{3}$ \\ ${ }^{1}$ Eng. Florestal, Dr., Depto. de Ciências Florestais, UFAM, Manaus, AM, Brasil - nspio@ufam.edu.br \\ ${ }^{2}$ Eng. Florestal, PhD., Depto. de Engenharia e Tecnologia Florestal, UFPR, Curitiba, PR, Brasil - skjockey@gmail.com; \\ setsuo@ufpr.br; mprocha@ufpr.br \\ ${ }^{3}$ Eng. Florestal, Dr., Depto. de Ciências Florestais, UFAM, Manaus, AM, Brasil - ulisses@ufam.edu.br; fercalu@ufam.edu.br
}

Recebido para publicação: 03/03/2010 - Aceito para publicação: 18/01/2011

\begin{abstract}
Resumo
O objetivo deste trabalho foi avaliar a resistência e elasticidade em flexão estática em painéis LVL produzidos com lâminas pré-classificadas de Eucalyptus grandis W. Hill ex Maiden de diferentes idades. Foram selecionadas 15 árvores, sendo oito com idade de 15 anos e sete com idade 20 anos, processadas em torno laminador e em seguida pré-classificadas não destrutivamente. Foram geradas três classes de rigidez para 15 anos e três para 20 anos, sendo os painéis produzidos dentro de cada classe. Para avaliar o confronto intraclasses de rigidez, foi realizada uma Anova a 95\% de probabilidade, e um teste de médias de Tukey a $95 \%$ de significância. Para o MOR foi observada uma relação direta entre as classes de rigidez, ou seja, com o aumento da classe houve uma resposta direta na variável. O efeito é observado tanto na idade de 15 quanto de 20 anos. O MOE teve comportamento semelhante à densidade dos painéis. Para a idade de 15 anos, existe relação direta entre as classes de rigidez e valores do MOE. Na idade de 20 anos não houve relação entre MOE e classe de rigidez. Assim, a propriedade avaliada indica a possibilidade de produção, em escala comercial, de painéis com características diferenciadas por classe de rigidez.
\end{abstract}

Palavras-chave: Stress wave timer; flexão estática; painéis LVL.

\begin{abstract}
Physical properties analysis of LVL panels produced with Eucalyptus grandis. This research was developed in order to evaluate the mechanical property of static bending of LVL panels produced from pre-classified veneers of Eucalyptus grandis Hill ex-Maiden differently aged by non-destructive method. Fifteen trees were selected for peeling, 8 were 15 years old and 7 were 20 years old, and preclassified by non destructive method. The pre-classifying process was applied to generate three stiffness grades of veneers from the 15 years old trees as well as three from the 20 years old ones, and the LVL was produced for each grade. Results for MOR revealed a direct relationship among the stiffness grade classes, it is, grades increasing results in direct effects on static bending strength for both ages of trees. There were similar correlation between MOE and density of LVL panels. For the LVL produced from 15 years old trees there was direct correlation between stiffness and MOE. On the other hand, for the ones from 20 years old trees there was no correlation between these variables. As result, the evaluated property points to production possibilities, commercial scale, and panels with different characteristics by stiffness classes.

Keywords: Stress wave timer; static bending; LVL panels.
\end{abstract}

\section{INTRODUÇÃO}

Os painéis LVL foram introduzidos no mercado como um produto alternativo para uso estrutural e possibilitaram o desenvolvimento de vigas com formato "I", denominadas de "I-joist", hoje amplamente empregadas nos Estados Unidos para montagem de estruturas de madeira. Sua principal vantagem é a economia no consumo de madeira e maior relação peso/resistência das peças (PEDROSA, 2003). 
A análise dos aspectos de qualidade e rigidez da madeira é fundamental para a classificação de peças destinadas a usos diversos, ou ainda para a avaliação de lâminas destinadas à fabricação de peças laminadas coladas. Essas avaliações são realizadas com base em especificações normatizadas para cada aplicação específica (GONÇALVES; PUCCINI, 1998).

No Brasil, essas normas são baseadas unicamente na avaliação visual que, em alguns casos, pode ser bastante falha, pois nem sempre é possível visualizar ou mensurar claramente aspectos ligados à qualidade e rigidez, como, por exemplo, a detecção de nós e ataques de insetos, entre outros defeitos, já que eles podem atingir proporções internas na peça impossíveis de serem vistas superficialmente.

Os métodos utilizados para classificar a madeira não destrutivamente possuem o objetivo de facilitar a especificação do material na fase de concepção do projeto estrutural, enquanto os métodos de classificação visual e mecânica são utilizados com a intenção de homogeneizar e selecionar lotes de madeira com características específicas, visando o adequado aproveitamento do material para fins estruturais (SALES, 1998).

Ensaios não destrutivos de madeira podem ser definidos como métodos utilizados para identificação das propriedades físicas e mecânicas de um material sem que haja o comprometimento de seu uso final (GABRIEL, 2000).

Diante do exposto, o presente estudo tem por objetivo avaliar a resistência e elasticidade em flexão estática em painéis LVL produzidos com lâminas pré-classificadas de Eucalyptus grandis W. Hill ex Maiden de diferentes idades (15 e 20 anos), com o auxílio do stress wave timer, e como hipótese a ser testada, a influência ou não da classe de rigidez no comportamento da propriedade avaliada.

\section{MATERIAL E MÉTODOS}

\section{Coleta do material}

Foram selecionadas 15 árvores de Eucalyptus grandis, sendo 8 com idade de 15 anos e 7 com idade de 20 anos, diâmetro mínimo de $40 \mathrm{~cm}$, troncos cilíndricos livres de bifurcações, com alturas variáveis, provenientes da Klabin Fabricadora de Papel e Celulose, localizada na região de Telêmaco Borba (PR). A amostragem das árvores foi seletiva com base no melhor diâmetro para laminação.

\section{Determinação da densidade básica da madeira}

Discos de aproximadamente $5,0 \mathrm{~cm}$ de espessura foram retirados da base, meio e topo das árvores para análise da densidade básica das diferentes idades avaliadas. (1968).

A equação utilizada para a determinação foi a mesma de Tsoumis (1991) e Kollmann e Cotê

Um total de trinta discos, sendo quinze para a idade de 15 anos e quinze para a idade de 20 anos, foram utilizados para a determinação dessa propriedade.

Através de análise de variância a $95 \%$ de probabilidade, foram determinadas as diferenças estatísticas entre as idades.

\section{Processo de obtenção de lâminas}

Com as toras na indústria laminadora, foi realizado um cozimento (aquecimento a vapor), tendo como base a densidade da espécie, durante 32 horas a uma temperatura em torno de $80{ }^{\circ} \mathrm{C}$, a fim de tornar a madeira mais plástica e também na tentativa de aliviar as tensões de crescimento, pois as toras receberam novo destopo para ficar nas dimensões do torno laminador.

As espessuras das lâminas obtidas na laminação foram de 2,0 mm e de 2,6 mm, para toras de ambas as idades. A escolha se deu em função da maior facilidade de obtenção e devido ao padrão utilizado pela indústria, bem como em função de problemas associados à secagem do Eucalyptus spp. quando se utilizam lâminas com grandes espessuras.

\section{Classificação das lâminas}

Para avaliar efeito do módulo de elasticidade dinâmico sobre a espessura, foi realizada análise de variância seguida de teste de médias de Tukey, ambos a 95\% de probabilidade.

A classificação das lâminas foi baseada na avaliação não destrutiva através do Módulo de Elasticidade Dinâmico (MOED) individual das lâminas, sendo utilizada a metodologia adotada por Matos 
(1997), na qual o valor mínimo aceitável para o MOED comumente utilizado pelas indústrias do mercado americano para a produção de painéis LVL é de $140.614 \mathrm{kgf} / \mathrm{cm}^{2}$ (KRETSCHMANN et al., 1993). De posse dos resultados, foi estabelecido um limite mínimo de rigidez das lâminas para a produção dos painéis.

Foram estabelecidas as seguintes classes de rigidez, tendo como base o MOED mais uma amplitude de classe por idade, apresentadas na tabela 1.

As amplitudes de classe utilizadas nesta pesquisa foram duas: $20.000 \mathrm{kgf} / \mathrm{cm}^{2}$ para a idade de 15 anos e $30.000 \mathrm{kgf} / \mathrm{cm}^{2}$ para a idade de 20 anos, obedecendo a dois critérios básicos:

a) Critério de classes independentes e mutuamente exclusivas;

b) Critério arbitrário, para gerar e representar o melhor perfil da população avaliada.

Tabela 1. Classes de rigidez geradas pelo stress wave timer através de valores calculados pelo MOED.

Table 1. Grades of stiffness generated by stress wave timer based on dynamic modulus of elasticity values.

\begin{tabular}{lccc}
\hline $\begin{array}{l}\text { Idade } \\
\text { (anos) }\end{array}$ & $\begin{array}{c}\text { Espessura } \\
(\mathbf{m m})\end{array}$ & Classe de rigidez & $\begin{array}{c}\text { Amplitude } \\
\left(\mathbf{x 1 0 0 0} \mathbf{~ k g f} / \mathbf{c m}^{2}\right)\end{array}$ \\
\hline & 2,00 & NC & $<140$ \\
& & 1 & MOED +20 \\
15 & 2,60 & 3 & $<140$ \\
& & NC & MOED + 20 \\
& & 2 & $<170$ \\
20 & 2,00 & 3 & MOED + 30 \\
& & NC & $<170$ \\
& & 2 & MOED + 30 \\
\hline
\end{tabular}

As lâminas não classificadas também foram utilizadas na construção dos painéis, sendo posicionadas como miolo no centro da chapa. Sua utilização justifica-se pelo fato de o centro do painel ser a região de menor solicitação de esforços. das lâminas.

A figura 1 mostra o stress wave timer, equipamento utilizado para a pré-classificação individual

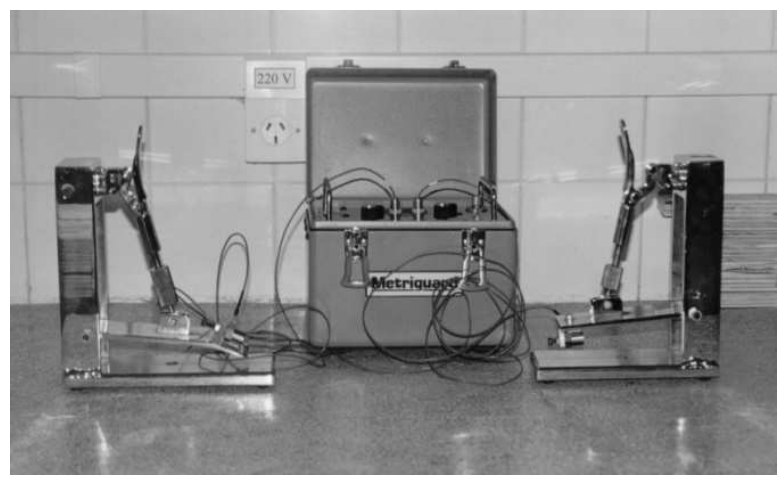

Figura 1. Equipamento utilizado na pré-classificação das lâminas - stress wave timer.

Figure 1. Equipment used for veneers pre-classifying - stress wave machine. 
A figura 2 apresenta o método utilizado para obtenção da rigidez das lâminas através do stress wave timer. Os transdutores foram posicionados no centro de cada lâmina avaliada.

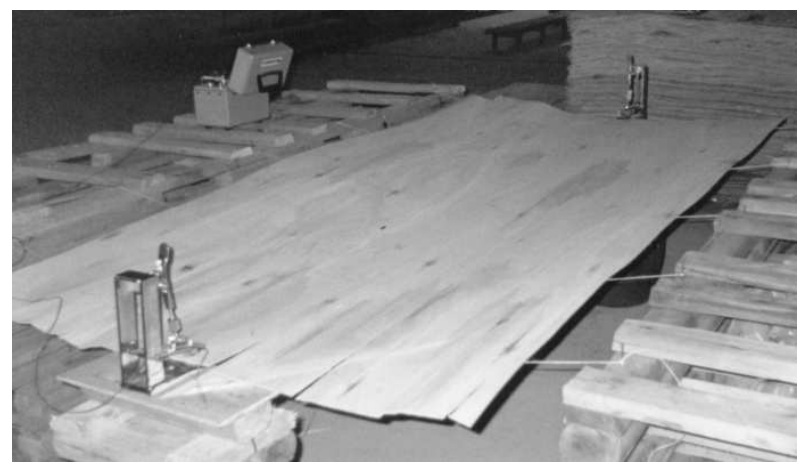

Figura 2. Obtenção da rigidez das lâminas e módulo de elasticidade dinâmico pelo stress wave timer. Figure 2. Obtention of veneers stiffness and dynamic modulus of elasticity by stress wave timer.

\section{Propriedades mecânicas das lâminas - método não destrutivo}

Foi utilizado um equipamento que verifica o tempo de propagação de uma onda de tensão através das lâminas (stress wave timer) (Figura 1), constituído de um sistema de garras, acelerômetros e um registrador de tempo. Foram tomados os tempos de propagação das ondas em microssegundos no sentido axial de cada lâmina mostrada na figura 2.

Todas as classes de rigidez propostas têm origem no módulo de elasticidade dinâmico, calculado para cada lâmina.

\section{Módulo de elasticidade dinâmico - MOED lâminas}

Esse cálculo foi feito a partir da equação utilizada por Matos (1997), fornecida pelo fabricante do equipamento através dos valores medidos individualmente para cada lâmina.

A equação utilizada é a que segue, (METRIGUARD, 1997):

$$
\text { MOEd }=\delta \times V^{2} \times\left(\frac{1}{g}\right)
$$

em que: $M O E d=$ módulo de elasticidade dinâmico $\left(\mathrm{kgf} / \mathrm{cm}^{2}\right)$;

$\delta=$ densidade da lâmina $\left(\mathrm{kg} / \mathrm{m}^{3}\right)$;

$V=$ velocidade da onda longitudinal $(\mathrm{cm} / \mathrm{s})$;

$g=$ aceleração da gravidade $\left(9,804 \mathrm{~m} / \mathrm{s}^{2}\right)$.

A velocidade de propagação da onda foi obtida pela seguinte equação:

$$
V=\frac{d}{t}
$$

em que: $V=$ velocidade da onda longitudinal $(\mathrm{cm} / \mathrm{s})$;

$d=$ distância entre os transdutores $(\mathrm{cm})$;

$t=$ tempo de propagação $(\mu \mathrm{s})$.

Calculados os valores individuais do módulo de elasticidade dinâmico para as lâminas, foi realizada a divisão em três classes de rigidez nas diferentes idades: lâminas com valores de MOED denominadas classificadas, e lâminas com MOED abaixo de $140.614 \mathrm{kgf} / \mathrm{cm}^{2}$, denominadas não classificadas (NC).

A determinação do valor mínimo para a classificação das lâminas por classes de rigidez foi feita após análise da distribuição de frequência dos valores do módulo de elasticidade dinâmico.

\section{Densidade das lâminas}

Essa análise foi constituída pela pesagem individual de cada uma das lâminas e determinação do volume através da espessura, largura e comprimento em pontos distintos da lâmina. 
Para a determinação da densidade aparente das lâminas, a um teor de umidade em torno de $6 \%$, utilizou-se a equação comumente adotada para obtenção dessa propriedade:

$$
\delta=\frac{P}{V}
$$

em que: $\delta=$ densidade aparente $(6 \%)$, em $\left(\mathrm{g} / \mathrm{cm}^{3}\right)$;

$\mathrm{P}=$ peso seco da lâmina $(6 \%)$, em $(\mathrm{g})$;

$V=$ volume seco da lâmina $(6 \%), \mathrm{em}\left(\mathrm{cm}^{3}\right)$.

\section{Análise do MOED, idade e espessura das lâminas}

Foi proposto o modelo de análise apresentado na tabela 2 para a interpretação dos resultados, que também poderá ser adotado em futuras investigações dessa natureza.

Tabela 2. Modelo para confronto entre classes de rigidez $\left(\mathrm{kgf} / \mathrm{cm}^{2}\right)$ por fator.

Table 2. Comparison model between stiffness grades $\left(\mathrm{kgf} / \mathrm{cm}^{2}\right)$ by factor.

\begin{tabular}{lcccc}
\hline \multirow{2}{*}{ Fator } & \multicolumn{5}{c}{ MOED - Classes de Rigidez $\left(\mathbf{k g f} / \mathbf{c m}^{\mathbf{2}}\right) \mathbf{x ~ 1 0 0 0}$} \\
\cline { 2 - 5 } & $\mathbf{1}$ & $\mathbf{2}$ & $\mathbf{3}$ & $\mathbf{4}$ \\
\hline A $(15 / 2,0) 1$ & A1 & A2 & A3 & A4 \\
B $(15 / 2,6) 2$ & B1 & B2 & B3 & B4 \\
C $(20 / 2,0) 3$ & C1 & C2 34 & C4 \\
D $(20 / 2,6) 4$ & D1 & D2 & D3 & D4 \\
\hline
\end{tabular}

em que: $\mathrm{A}(15 / 2,0)=$ Idade 15 anos e Espessura 2,0 $\mathrm{mm}$.

Fator/Classes: $1=141$ a $160 ; 2=161$ a $180 ; 3=181$ a $200 ; 4$ = não classificada $(<140)$.

$\mathrm{B}(15 / 2,6)=$ Idade 15 anos e Espessura 2,6 mm.

Fator/Classes: 1 = 141 a $160 ; 2=161$ a $180 ; 3=181$ a $200 ; 4$ = não classificada $(<140)$.

$\mathrm{C}(20 / 2,0)=$ Idade 20 anos e Espessura 2,0 $\mathrm{mm}$.

Fator/Classes: $1=170$ a 200; $2=201$ a 230; $3=231$ a 260; 4 = não classificada $(<170)$.

D $(20 / 2,6)=$ Idade 20 anos e Espessura 2,6 mm.

Fator/Classes: $1=170$ a 200; $2=201$ a 230; $3=231$ a 260; 4 = não classificada $(<170)$.

Foi realizado um confronto entre as classes de rigidez em diferentes idades e espessura de lâmina, que serviu de base para a montagem dos painéis.

A interação dos fatores idade e espessura (tratamentos) ocorreu da seguinte maneira: AxB: confronto da espessura 2,0 versus a espessura 2,6 na idade de 15 anos; CxD: confronto da espessura 2,0 versus a espessura 2,6 na idade de 20 anos; AxC: confronto da idade 15 versus a idade 20 na espessura de 2,0; BxD: confronto da idade 15 versus a idade 20 na espessura de 2,6.

Para avaliar o confronto intraclasses de rigidez, foi realizada uma Anova a 95\% de probabilidade. Após análise de variância, foi processado um teste de médias de Tukey, a 95\% de significância, aplicado todas as vezes que a hipótese de nulidade era rejeitada.

\section{Produção dos painéis LVL}

Seu princípio de construção difere do painel compensado multilaminado, pela disposição das lâminas na mesma direção e não a da laminação cruzada. Dessa forma, a resistência do painel na direção longitudinal será maior, possibilitando seu uso em aplicações que requeiram maior resistência à flexão estática (TSOUMIS, 1991).

Os LVL produzidos nesta pesquisa foram compostos de 13 lâminas combinadas nas diferentes espessuras, com dimensões de 2,44 m x 1,10 m x 0,0254 m. Foram produzidos 36 painéis distribuídos por seis tratamentos. Entende-se por tratamentos as classes de rigidez obtidas pelo módulo de elasticidade dinâmico das lâminas.

A tabela 4 apresenta a posição das lâminas na produção dos painéis LVL por classe de rigidez e idade. 
Tabela 4. Posição das lâminas na produção dos LVL.

Table 4. Veneers positioning in the LVL production.

\begin{tabular}{|c|c|c|c|c|c|}
\hline $\begin{array}{l}\text { Idade } \\
\text { (anos) }\end{array}$ & $\begin{array}{c}\text { Classe de } \\
\text { rigidez }\end{array}$ & $\begin{array}{c}\text { Espessura } \\
\text { lâmina }(\mathbf{m m})\end{array}$ & $\begin{array}{c}\text { Número de } \\
\text { lâminas por painel }\end{array}$ & $\begin{array}{c}\text { Disposição das lâminas } \\
\text { no painel }\end{array}$ & $\begin{array}{c}\text { Espessura } \\
\text { do painel }(\mathbf{m m})\end{array}$ \\
\hline \multirow{3}{*}{15} & 1 & $2,0-2,6-n c$ & $8-4-1$ & $2_{(2,6)^{-}}-4_{(2,0)}-1_{(\mathrm{nc})^{-}}-4_{(2,0)}-2_{(2,6)}$ & \multirow{3}{*}{29} \\
\hline & 2 & $2,0-2,6-n c$ & $8-4-1$ & $2_{(2,6)^{-}}-4_{(2,0)}-1_{(\mathrm{nc})^{-}}-4_{(2,0)}-2_{(2,6)}$ & \\
\hline & 3 & $2,0-2,6-n c$ & $8-4-1$ & $2_{(2,6)^{-}}-4_{(2,0)}-1_{(n c)}-4_{(2,0)}-2_{(2,6)}$ & \\
\hline \multirow{3}{*}{20} & 4 & $2,0-2,6-\mathrm{nc}$ & $8-4-1$ & $2_{(2,6)^{-}}-{ }_{(2,0)}-1_{(\mathrm{nc})}-4_{(2,0)}-2_{(2,6)}$ & \multirow{3}{*}{29} \\
\hline & 5 & $2,0-2,6-n c$ & $8-4-1$ & $2_{(2,6)^{-}}-4_{(2,0)}-1_{(n c)^{-}}-4_{(2,0)}-2_{(2,6)}$ & \\
\hline & 6 & $2,0-2,6-n c$ & $8-4-1$ & $2_{(2,6)}-4_{(2,0)}-1_{(n c)}-4_{(2,0)}-2_{(2,6)}$ & \\
\hline
\end{tabular}

NC: Lâmina não classificada.

Os painéis produzidos nesta pesquisa seguiram a pré-classificação das lâminas, que foram agrupadas nas classes preestabelecidas, como mostra a tabela 5.

Tabela 5. Distribuição dos painéis LVL por idade e classe de rigidez.

Table 5. Distribution of LVL panels by ages and stiffness grades.

\begin{tabular}{lcccccc}
\hline Idade & \multicolumn{7}{c}{ MOED lâminas } \\
\cline { 2 - 7 }$($ anos $)$ & $\mathbf{1 4 1 - 1 6 0}$ & $\mathbf{1 6 1 - 1 8 0}$ & $\mathbf{1 8 1 - 2 0 0}$ & $\mathbf{1 7 0 - 2 0 0}$ & $\mathbf{2 0 1 - 2 3 0}$ & $\mathbf{2 3 1 - 2 6 0}$ \\
\hline 15 & 6 & 6 & 6 & - & - & - \\
20 & - & - & - & 6 & 6 & 6 \\
\hline
\end{tabular}

\section{Ciclo de prensagem}

O ciclo de prensagem utilizado para o experimento foi elaborado de acordo com orientação técnica fornecida pelo fabricante da resina, bem como seguindo critérios técnicos empregados na própria indústria, sendo apresentado na tabela 6.

Tabela 6. Ciclo de prensagem.

Table 6. Press cycle.

\begin{tabular}{ll}
\hline Variável & Valores \\
\hline Adesivo & À base de resina fenol-formaldeído \\
Temperatura & $150{ }^{0} \mathrm{C}$ \\
Tempo de prensagem & $1 \mathrm{~min} / \mathrm{mm}$ \\
Gramatura de cola & $350 \mathrm{~g} / \mathrm{m}^{2}$ \\
Pressão específica & $12 \mathrm{kgf} / \mathrm{cm}^{2}$ \\
Umidade lâmina & $4-8 \%$ \\
\hline Fonte: Indústria de Compensado Triângulo Ltda.
\end{tabular}

A resina utilizada foi à base de fenol-formaldeído. A gramatura de cola por linha dupla foi de $350 \mathrm{~g} / \mathrm{m}^{2}$. O preparo do adesivo se constituiu basicamente da mistura da resina, em uma batedeira, com as demais partes componentes da formulação, farinha de trigo, água, casca de noz em pó, também indicados pelo fabricante.

\section{RESULTADOS E DISCUSSÃO}

\section{Análise da densidade dos painéis}

A tabela 7 apresenta os valores da densidade analisada por idade e classe de rigidez, bem como as estatísticas descritas para o ensaio.

Ao se comparar a densidade entre as classes de rigidez e idades, a tabela 7 revela que para o Eucalyptus grandis 15 anos as três classes de rigidez apresentam diferenças estatísticas significativas ao nível de $95 \%$ de probabilidade, confirmando resultados similares obtidos na pré-classificação das lâminas. 
Tabela 7. Valores médios da densidade dos painéis por idade e classe de rigidez.

Table 7. Average values of board densities by ages and stiffness grades.

\begin{tabular}{lccccccc}
\hline $\begin{array}{l}\text { Idade } \\
(\text { anos })\end{array}$ & C.R. & $\begin{array}{c}\text { Densidade } \\
\left(\mathbf{g} / \mathbf{c m}^{\mathbf{3}}\right)\end{array}$ & $\begin{array}{c}\text { Valor } \\
\text { mínimo }\end{array}$ & $\begin{array}{c}\text { Valor } \\
\text { máximo }\end{array}$ & $\begin{array}{c}\text { T.U. } \\
\mathbf{\%}\end{array}$ & $\begin{array}{c}\text { Desvio } \\
\text { padrão }\end{array}$ & $\begin{array}{c}\text { C.V. } \\
\text { \% }\end{array}$ \\
\hline \multirow{3}{*}{15} & 1 & $0,6353 \mathrm{~A}$ & 0,6051 & 0,6600 & 8,98 & 0,018552 & 2,92 \\
& 2 & $0,6664 \mathrm{~B}$ & 0,6438 & 0,6841 & 8,98 & 0,012049 & 1,80 \\
& 3 & $0,7003 \mathrm{C}$ & 0,6896 & 0,7063 & 8,94 & 0,005898 & 0,84 \\
& 4 & $0,7728 \mathrm{D}$ & 0,7538 & 0,7971 & 9,47 & 0,014582 & 1,88 \\
& 5 & $0,7766 \mathrm{D}$ & 0,7417 & 0,8277 & 9,09 & 0,023643 & 3,02 \\
& 6 & $0,7836 \mathrm{D}$ & 0,7517 & 0,7953 & 9,35 & 0,012712 & 1,64 \\
\hline
\end{tabular}

Letras diferentes denotam diferenças estatísticas ao nível de 95\% de probabilidade; Teste de média: Tukey; CR: Classe de rigidez; T.U.: Teor de umidade médio do ensaio (\%); CV: Coeficiente de variação (\%).

Para o Eucalyptus grandis 20 anos, verifica-se um comportamento diferenciado quando comparado à idade anterior. Nessa idade, 20 anos, as médias encontradas por classe de rigidez não diferiram estatisticamente ao nível de $95 \%$ de probabilidade, e os valores numéricos para a densidade encontram-se bem próximos, podendo-se ressaltar o baixo coeficiente de variação encontrado para os tratamentos, indicando pouca variação entre os valores observados dentro dos tratamentos.

Essa faixa de valores médios bem próximos para a densidade na idade 20 anos pode ser um indicador de baixo contraste na estrutura anatômica da espécie, bem como presença de maiores proporções do lenho adulto ao longo do fuste.

\section{Módulo de ruptura na flexão estática - MOR}

A análise de variância realizada entre as classes de rigidez para o módulo de ruptura no ensaio de flexão estática e revela haver diferenças significativas entre as classes de rigidez, por idade.

A tabela 8 apresenta os valores médios para o ensaio de flexão estática, módulo de ruptura obtido por idade para todas as classes de rigidez e a comparação estatística desses valores, assim como as estatísticas descritivas para o ensaio.

Tabela 8. Valores médios para o ensaio de flexão estática módulo de ruptura dos painéis em diferentes idades e classes de rigidez.

Table 8. Average values of modulus of rupture in static bending tests for panels with differents ages and stiffness grades.

\begin{tabular}{lccccccc}
\hline $\begin{array}{l}\text { Idade } \\
\text { (anos) }\end{array}$ & C.R. & $\begin{array}{c}\text { MOR } \\
\left(\mathbf{k g f} / \mathbf{c m}^{\mathbf{2}}\right)\end{array}$ & $\begin{array}{c}\text { Valor } \\
\text { mínimo }\end{array}$ & $\begin{array}{c}\text { Valor } \\
\text { máximo }\end{array}$ & $\begin{array}{c}\text { T.U. } \\
\text { \% }\end{array}$ & $\begin{array}{c}\text { Desvio } \\
\text { padrão }\end{array}$ & $\begin{array}{c}\text { C.V. } \\
\text { \% }\end{array}$ \\
\hline \multirow{3}{*}{15} & 1 & $890,570 \mathrm{~A}$ & 688,201 & 1032,300 & 8,98 & 120,903 & 13,57 \\
& 2 & $992,389 \mathrm{AB}$ & 803,522 & 1130,880 & 8,98 & 95,570 & 9,63 \\
& 3 & $1049,970 \mathrm{~B}$ & 822,122 & 1184,820 & 8,94 & 107,522 & 10,24 \\
& 4 & $1138,790 \mathrm{~B}$ & 989,522 & 1296,420 & 9,47 & 106,903 & 9,38 \\
& 5 & $1322,790 \mathrm{C}$ & 1008,120 & 1566,120 & 9,09 & 167,585 & 12,67 \\
& 6 & $1416,580 \mathrm{C}$ & 1162,500 & 1566,12 & 9,35 & 129,761 & 9,16 \\
\hline
\end{tabular}

Letras diferentes denotam diferenças estatísticas ao nível de 95\% de probabilidade; Teste de média: Tukey; C.R.: Classe de Rigidez; MOR- Módulo de ruptura na flexão estática; T.U.: Teor de umidade médio do ensaio (\%); C.V.: Coeficiente de variação (\%).

Através do teste de Tukey, foram identificadas diferenças significativas para os valores médios do MOR entre as classes de rigidez por idades analisadas. Isso mostra que o efeito da pré-classificação foi significativo sobre o MOR, gerando valores diferenciados. A tabela 8 também revela um comportamento semelhante para as duas idades. As duas classes de rigidez mais elevadas não apresentaram diferenças estatísticas significativas para 15 e 20 anos.

Do ponto de vista prático, pode-se considerar que, apesar da falta de significância estatística entre os valores das classes de rigidez mais elevadas, isso não inviabiliza o uso do pré-classificador.

É necessário considerar que na idade 20 anos os valores médios para a densidade não apresentaram diferenças estatísticas significantes, o que leva a presumir que o MOR esteja sendo influenciado por esse comportamento da variável citada. 
Na idade 15 anos, supõe-se que pode ter havido influência da própria composição da estrutura anatômica da espécie sobre o MOR, aliada à elevada presença de nós naturais da madeira nas lâminas que compõem os painéis, fato que foi bem observado durante a laminação e montagem dos painéis.

Comparando os valores médios com a literatura existente, Matos (1997), trabalhando com painéis LVL de Pinus taeda, determinou valores mais baixos, em média $374,0 \mathrm{kgf} / \mathrm{cm}^{2}$, sendo importante observar a diferença existente entre as massas específicas dos painéis.

\section{Tensão no limite proporcional - TLP}

Os resultados da análise de variância realizada entre as classes de rigidez para o módulo de elasticidade no ensaio de flexão estática revela haver diferenças significativas entre as classes de rigidez por idades analisadas. A tabela 9 apresenta os valores médios para o ensaio de flexão estática, tensão no limite proporcional obtido por idade para todas as classes de rigidez e a comparação estatística desses valores, assim como as estatísticas descritivas.

Tabela 9. Valores médios para o ensaio de flexão estática tensão no limite proporcional dos painéis em diferentes idades e classes de rigidez (C.R.).

Table 9. Average values of tension at proportional limit in static bending tests for panels with differents ages and stiffness grades.

\begin{tabular}{lccccccc}
\hline $\begin{array}{l}\text { Idade } \\
\text { (anos) }\end{array}$ & C.R. & $\begin{array}{c}\text { TLP } \\
\left(\mathbf{k g f} / \mathbf{c m}^{2}\right)\end{array}$ & $\begin{array}{c}\text { Valor } \\
\text { mínimo }\end{array}$ & $\begin{array}{c}\text { Valor } \\
\text { máximo }\end{array}$ & $\begin{array}{c}\text { T.U. } \\
\text { \% }\end{array}$ & $\begin{array}{c}\text { Desvio } \\
\text { padrão }\end{array}$ & $\begin{array}{c}\text { C.V. } \\
\text { \% }\end{array}$ \\
\hline & 1 & $458,97 \mathrm{~A}$ & 398,863 & 545,284 & 8,98 & 35,1252 & 7,65 \\
15 & 2 & $461,09 \mathrm{AB}$ & 428,697 & 517,688 & 8,98 & 26,0244 & 5,64 \\
& 3 & $471,82 \mathrm{AB}$ & 440,991 & 497,930 & 8,94 & 20,5431 & 4,35 \\
& 4 & $481,49 \mathrm{AB}$ & 450,224 & 507,746 & 9,47 & 21,5096 & 4,46 \\
20 & 5 & $484,04 \mathrm{AB}$ & 418,103 & 543,736 & 9,09 & 39,3340 & 8,12 \\
& 6 & $491,54 \mathrm{~B}$ & 434,157 & 539,188 & 9,35 & 25,1358 & 5,11 \\
\hline
\end{tabular}

Letras diferentes denotam diferenças estatísticas ao nível de 95\% de probabilidade; Teste de média: Tukey. C.R.: Classe de rigidez; TLP: Tensão no limite proporcional; T.U.: Teor de umidade médio do ensaio (\%); CV: Coeficiente de variação (\%).

Pelo teste de Tukey, tabela 9, foram identificadas diferenças significativas para os valores médios da TLP entre as classes de rigidez por idades analisadas. Isso mostra que o efeito da préclassificação foi significativo sobre a TLP apenas para o fator idade.

Na idade 20 anos é possível observar os valores médios por classe de rigidez e comparar com os valores obtidos na densidade (Tabela 7) e com isso supor que haja uma boa correlação entre as varáveis, devido ao comportamento similar entre ambas. A tabela 9 revela também que apenas a classes $1^{\underline{a}}$ na idade 15 anos e a classe $6^{\underline{a}}\left(3^{\underline{a}}\right.$ classe de rigidez) são diferentes estatisticamente.

Matos (1997), trabalhando com painéis LVL de Pinus taeda, determinou valores para a tensão no limite proporcional inferiores, em média 50\%, aos encontrados nesta pesquisa, em torno de $211 \mathrm{kgf} / \mathrm{cm}^{2}$. Isso pode ser explicado pela diferença existente entre espécies. Os valores determinados pelo citado autor tornam-se importantes, pois indicam coerência nos resultados aqui determinados.

\section{Módulo de elasticidade - MOE}

Os resultados da análise de variância realizada entre as classes de rigidez para o MOE no ensaio de flexão estática revela haver diferenças significativas entre as classes de rigidez analisadas, por idade.

A tabela 10 apresenta os valores médios para o ensaio de flexão estática, módulo de elasticidade obtido por idade para todas as classes de rigidez e a comparação estatística desses valores, assim como as estatísticas descritivas para o ensaio.

Através do teste de Tukey, tabela 10, foram identificadas diferenças significativas para os valores médios do MOE entre as classes de rigidez por idades analisadas. Isso mostra que o efeito da pré-classificação, para algumas classes de rigidez, foi significativo sobre o MOE, gerando resultados diferenciados.

Outro aspecto interessante observado na tabela 10 foi a baixa variação para o MOE, que não respeitou, em alguns casos, as classes de rigidez pré-estabelecidas. Isso é marcante quando se observa a ausência de diferenças estatísticas entre classes de rigidez em diferentes idades. Também chama a atenção que dentro da idade de 20 anos as duas maiores classes de rigidez não diferem estatisticamente. 
Tabela 10. Valores médios para o ensaio de flexão estática módulo de elasticidade dos painéis em diferentes idades e classes de rigidez.

Table 10. Average values for the static bending modulus test for panels with differents ages and stiffness grades.

\begin{tabular}{lccccccc}
\hline $\begin{array}{l}\text { Idade } \\
(\text { anos) }\end{array}$ & C.R. & $\begin{array}{c}\text { MOE } \\
\left(\mathbf{k g f} / \mathbf{c m}^{2}\right)\end{array}$ & $\begin{array}{c}\text { Valor } \\
\text { mínimo }\end{array}$ & $\begin{array}{c}\text { Valor } \\
\text { máximo }\end{array}$ & $\begin{array}{c}\text { T.U. } \\
\text { \% }\end{array}$ & $\begin{array}{c}\text { Desvio } \\
\text { padrão }\end{array}$ & $\begin{array}{c}\text { C.V. } \\
\text { \% }\end{array}$ \\
\hline \multirow{3}{*}{15} & 1 & 94110,5 A & 85881,2 & 98827,2 & 8,98 & 3704,22 & 3,93 \\
& 2 & 96326,6 B & 94054,1 & 98984,4 & 8,98 & 1249,45 & 5,29 \\
& 3 & 97628,6 BC & 95821,6 & 101212,0 & 8,94 & 1670,59 & 7,71 \\
20 & 4 & 98842,9 BC & 95235,1 & 106152,0 & 9,47 & 3402,37 & 3,44 \\
& 5 & 99044,6 C & 97071,4 & 103544,0 & 9,09 & 1953,55 & 4,97 \\
& 6 & $99096,1 \mathrm{C}$ & 94327,0 & 103462,0 & 9,35 & 2244,15 & 5,26 \\
\hline
\end{tabular}

Letras diferentes denotam diferenças estatísticas ao nível de 95\% de probabilidade; Teste de média: Tukey. C.R.: Classe de rigidez; MOE: Módulo de elasticidade na flexão estática; T.U.: Teor de umidade médio do ensaio (\%); CV: Coeficiente de variação (\%).

Pode-se acrescentar a essa análise que os valores encontrados para o MOE por classe de rigidez e idade possam ser reflexos da influência da densidade dos painéis.

Para Tsoumis (1991), citado por Bortoletto Júnior (2000), a elasticidade é a propriedade de um corpo de retornar à sua condição inicial (dimensão e forma) quando a carga que gerou a tensão e a deformação for removida. Isso ocorre abaixo do limite de proporcionalidade. Se o carregamento é continuado acima desse limite, parte da deformação é permanente. A relação entre tensão e deformação define o Módulo de Elasticidade (Young's Modulus), válido somente até o limite proporcional, parâmetro indicativo da rigidez do material. Quanto maior o MOE, mais rígido o material, ou seja, maior é a sua capacidade de suportar altas tensões sem grandes deformações.

Matos (1997), trabalhando com painéis estruturais LVL de Pinus taeda, determinou valores para o MOE em torno de $96.726 \mathrm{kgf} / \mathrm{cm}^{2}$ para painéis com lâminas de $3,2 \mathrm{~mm}$ de espessura, $92.083 \mathrm{kgf} / \mathrm{cm}^{2}$ para painéis com lâminas de $4,2 \mathrm{~mm}$ e de $73.200 \mathrm{kgf} / \mathrm{cm}^{2}$ para madeira sólida da mesma espécie. Com isso, os resultados encontrados para 15 e 20 anos, neste estudo, ratificam a elevada rigidez da madeira analisada e na prática torna-se indicador de qualidade do produto.

\section{CONCLUSÕES}

- A propriedade mecânica avaliada indica a possibilidade de produção e comercialização de painéis com propriedades diferenciadas por classe de resistência.

- Na utilização dos painéis, a idade 20 anos é mais indicada para uso estrutural, o que não descarta a menor idade.

- As classes de rigidez propostas indicam comportamento diferenciado em relação à elasticidade dos painéis, tanto na idade 15 quanto na idade 20 anos, confirmando a hipótese avaliada neste artigo.

\section{REFERÊNCIAS}

BORTOLETTO JÚNIOR, G. Determinação do módulo de elasticidade na flexão estática pelo uso do deflectômetro e do sensor de deslocamento em ensaios não destrutivos. In: Anais do VII Encontro Brasileiro em Madeiras e em Estruturas de Madeira (arquivo de computador, 192). São Carlos-SP: EESC/USP, 2000. 1 Cd-room.

GABRIEL, M. S. C. O uso das ondas de tensão na qualificação mecânica da madeira serrada de Pinus taeda L. Botucatu 2000, 94 f. Dissertação (Mestrado em Agronomia) - Faculdade de Ciências Agrárias, Universidade Estadual Paulista - Campus de Botucatu.

GONÇALVES, R.; PUCCINI, C. T. Utilização do ultrassom na avaliação de aspectos de qualidade da madeira. In: ENCONTRO BRASILEIRO EM MADEIRAS E EM ESTRUTURAS DE MADEIRAS, 6. Florianópolis, 1998. Anais.

KOLlmanN, F. F. P.; CÔTÉ JR., W. A. Principles of wood science and technology: solid wood. Berlin: Springer-Verlag, 1968. 562 p. 
KRETSCHMANN, D. E.; MOODY, R. C.; PELLERIN, R. F.; BENDTSEN, B. A.; CAHILl, J. M.; MCALISTER, R. H.; SHAR, D. W. Effect of various proportions of juvenile wood on laminated veneer lumber. USDA. Forest Service. Research Paper, FPL-521, Madison, p. 1 - 31, 1993.

MATOS, J. L. M. Estudo sobre a produção de painéis estruturais de lâminas paralelas de Pinus taeda L. Curitiba 1997, 117 p. Tese (Doutorado em Ciências Florestais) - Universidade Federal do Paraná.

METRIGUARD INC. Metriguard Model $239^{\mathrm{A}}$ Stress Wave Timer. Manual care and instructions, theory and data reduction. Pullman, 1997.

PEDROSA, A. L. Desempenho estrutural de vigas "I" constituídas por PLP e compensado de Pinus taeda L. e Eucalyptus dunnii Maiden e OSB de Pinus spp. 104 p. Dissertação (Mestrado em Ciências Florestais) - Universidade Federal do Paraná, Curitiba, 2003.

SALES, A. Metodologia para a obtenção dos sistemas de classes de resistência de madeira. In: VI EBRAMEM - Encontro Brasileiro em Madeiras e em Estruturas de Madeira. Florianópolis, SC. v. 1, p. 13 - 21. 1998.

TSOUMIS, G. Science and technology of wood, structure, properties, utilization. New York: Chapman e Hall, 1991. 498 p. 